\title{
APPLICATION OF AUTOREGRESSIVE EXTRAPOLATION TO THE CROSS-BOREHOLE TOMOGRAPHY
}

\author{
R.L. NOWACK AND C. LI
}

Department of Earth and Atmospheric Sciences, Purdue University, 550 Stadium Mall Dr., West Lafayette, IN 47907-1397, USA (nowack@purdue.edu, cli0713@gmail.com)

Received: September 29, 2005; Revised: February 2, 2006; Accepted: February 21, 2006

\begin{abstract}
Autoregressive (AR) extrapolation of travel-time data is tested using several synthetic tomography examples using a cross-borehole geometry. Earlier studies have shown crossborehole tomography using travel-times can have reduced resolution because of the limitations on ray coverage. We apply AR extrapolation to partial travel-time data and then compare the tomographic inversions using the full data and the extrapolated data. Both the overall patterns of the extended data and the tomographic reconstructions with the extended data show that AR extrapolation can effectively extend the synthetic crossborehole tomographic data to a broader coverage and can improve the cross-hole reconstruction images.
\end{abstract}

Key words: geophysical inversion, data regularization

\section{INTRODUCTION}

The ray coverage in cross-borehole tomography problems is inherently incomplete since there is not a completely surrounding coverage of sources and receivers. To improve the resolution of tomographic reconstructions using this geometry, we use two-stage autoregressive extrapolation (AR) techniques to extend the existing ray coverage to a broader range. Two-stage AR extrapolation algorithm is implemented based on the work of Claerbout (1992,1998), Claerbout and Fomel (2005) and Fomel and Claerbout (2003). $\mathrm{Li}$ and Nowack (2004) applied AR extrapolation methods to synthetic and real travel-time data for laboratory rock samples and showed that AR extrapolation techniques are effective in doing data extrapolation for seismic tomography experiments. Li and Nowack (2005) investigated travel-time surfaces for the laboratory geometry in order to perform quality control of the travel-time data. However, because of the circular array geometry used for experiments in the laboratory, the ray coverage for this geometry is often reasonably good even with partial ray coverage. It is therefore of interest to test the autoregressive extrapolation techniques using other experimental geometries. 
In this paper, we investigate autoregressive methods for travel-times using the crossborehole geometry. In this geometry, sources are located in one borehole and receivers are placed in a second borehole at some distance from the first. Because the source and receiver arrays are of limited length in each of the boreholes, this results in limited ray coverage for heterogeneities between the two boreholes. This could be partly corrected by including surface sources, but the source and receiver geometry would still be open at the bottom. The two-stage AR extrapolation method of Li and Nowack (2004) is applied to the partial travel-time data for the cross-borehole geometry, and tomographic reconstructions using the full and extrapolated datasets are then performed.

\section{AUTOREGRESSIVE METHODS}

Autoregressive extrapolation (AR) methods were originally applied in signal processing to predict time series and to perform prediction error filtering. AR extrapolation can also be used to replace missing or corrupted samples with estimates of their true samples. An autoregressive process of order $p$ can be written in the form

$$
y_{i}=\sum_{k=1}^{p} g_{k} y_{i-k}+\varepsilon_{i}=\bar{y}_{i}+\varepsilon_{i},
$$

where $g_{k}$ are the elements of the optimal predictor, $\bar{y}_{i}$ is the best linear mean-square predictor of $y_{i}$ based only on the previous $p$ samples and $\varepsilon_{i}$ is the prediction error. Autoregressive methods can also be applied for prediction and extrapolation of data in 1-D or higher dimensions (Claerbout and Fomel, 2005).

In the two-stage approach to autoregressive extrapolation, the first stage is to find the optimal prediction error (PE) filter from the known data and the second stage is to find the missing data using the resulting PE filter. The output of the PE filter tends to be white and the missing data values are estimated with the same spectrum as the known data. A geophysical inverse problem with both missing data and an unknown PE filter can be written

$$
\mathbf{A} x-\left[\begin{array}{l}
y_{a} \\
y_{b}
\end{array}\right]=r_{1} \approx 0
$$

and

$$
F\left[\begin{array}{l}
y_{a} \\
y_{b}
\end{array}\right]=r_{2} \approx 0 .
$$

In the Eq.(2a) the model parameters $x$ are related to the data by the sensitivity matrix $\mathbf{A}$, where $y_{a}$ are the known data and $y_{b}$ are the unknown data. The general fitting goal of setting $r_{1} \approx 0$ is equivalent to minimizing the data residuals. Using an $L_{2}$ norm, this results in the best fitting model that minimizes the residuals in a least squares sense. In Eq.(2b), the unknown data $y_{b}$ are related to the known data $y_{a}$ by the PE filter $F$. Setting 
$r_{2} \approx 0$ using a two-stage approach means first estimating the PEF filter $F$ from the known data $y_{a}$ and then estimating $y_{b}$. Eq.(2b) can then be solved as

$$
\left[\begin{array}{l}
A_{a} \\
A_{b}
\end{array}\right] x \sim\left[\begin{array}{l}
y_{a} \\
y_{b}^{\prime}
\end{array}\right],
$$

where $y_{b}^{\prime}$ is the autoregressive extrapolation of $y_{b}$ given $y_{a}$. The sensitivity matrix $\mathbf{A}$ is augmented by $A_{b}$ to account for the extrapolated data. This is a specialized but nonstandard form of the regularized inverse problem.

In 2-D AR extrapolation, the input and output arrays are 2-D arrays and the PE filter is also an array that is applied with the data. To use 1-D AR techniques for multidimensional geophysical problems, Claerbout (1998) proposed the concept of a helical filter. In this approach a multidimensional dataset is unwrapped into an effective 1-D dataset and then a 1-D AR algorithm is applied to perform the data extrapolation. For the seismic tomography problem, we have applied 2-D extrapolation of the travel-time data using the helical filter approach.
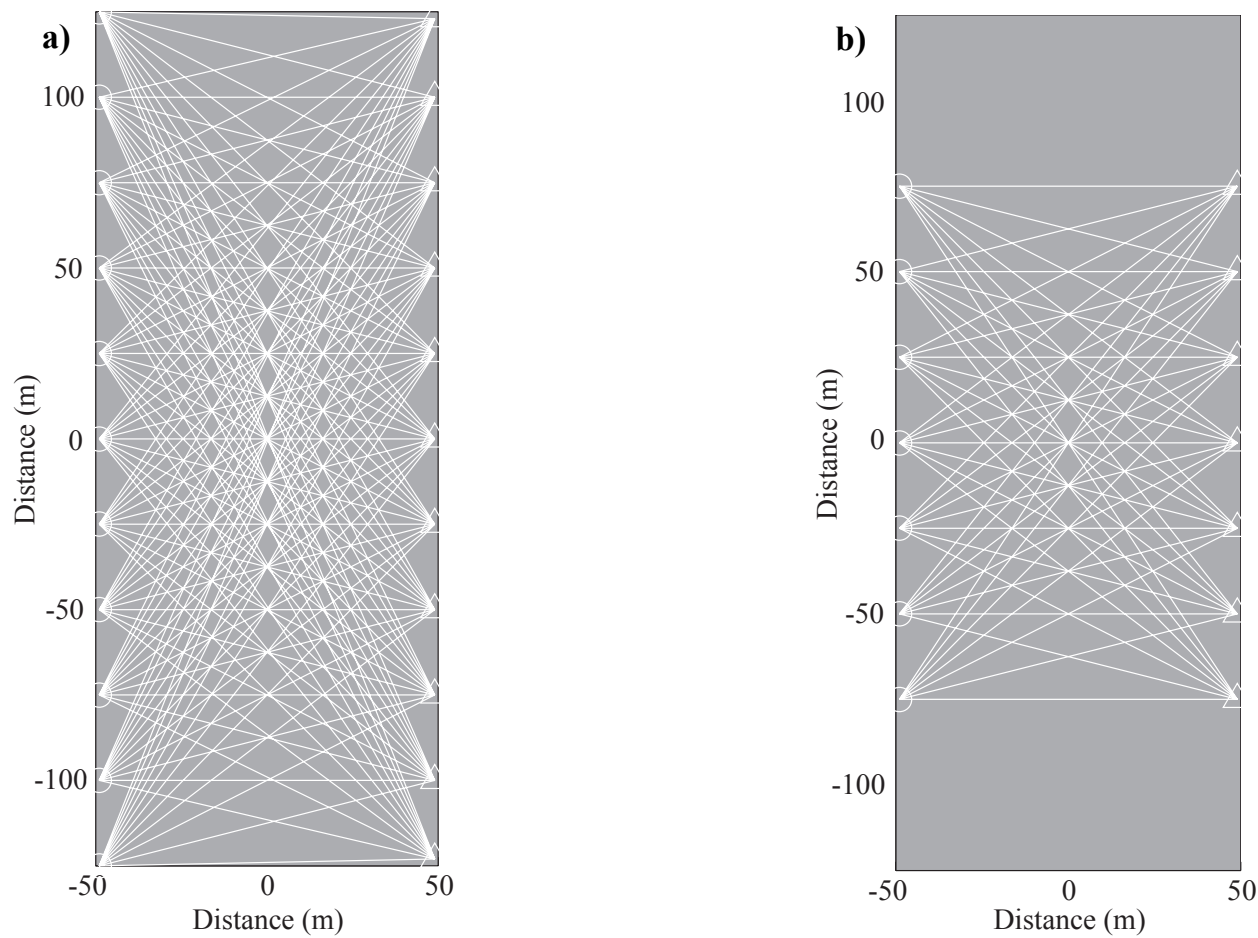

Fig. 1. a) Ray diagram for full ray coverage. A homogeneous starting model is used and the ray diagram for every five shots and receivers is shown. The shots are at the left edge of the model and the receivers are at the right edge. b) Ray diagram for partial ray coverage, after removing 10 shots and 10 receivers from the top and bottom of the boreholes. 
c)

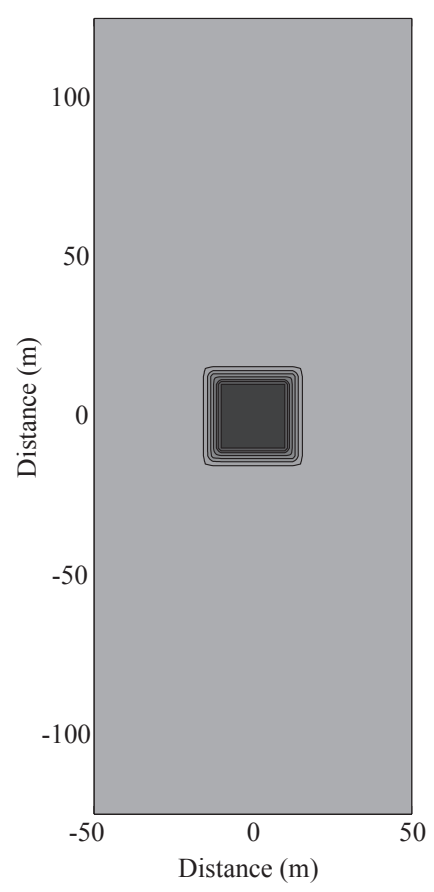

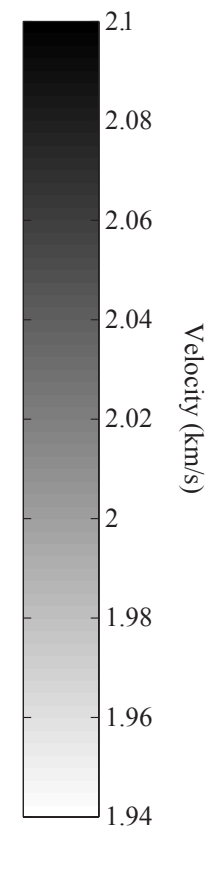

Fig. 1. c) True model for the single heterogeneity case. The square at the center shows a high velocity heterogeneity.

\section{CROSS-BOREHOLE EXPERIMENTAL GEOMETRY AND RAY COVERAGE}

We investigate seismic tomography using synthetic travel-time data with a crossborehole geometry and ray coverage. The size of the synthetic cross-borehole model is $100 \mathrm{~m}$ in width and $250 \mathrm{~m}$ in depth. There are a total of 51 shots and 51 receivers that are deployed along two separate boreholes. The spacing in the boreholes is $5 \mathrm{~m}$ for both shots and receivers. For each shot, rays are traced to every receiver and the travel-times are computed. Fig. 1a shows the homogeneous starting model with a velocity of $2.0 \mathrm{~km} / \mathrm{s}$ and a ray diagram showing every fifth shot and receiver point. The shot positions are on the left edge of the model and the receiver positions are on the right edge of the model. The partial ray coverage is shown in Fig. $1 \mathrm{~b}$ where 10 shots and 10 receivers from each end of the model have been removed. For both the full data and partial data, the ray coverage is denser at the center, and becomes sparser at the edges of the model. The true model for the first experiment is shown in Fig. 1c where the square at the center of the model is a higher velocity heterogeneity with a velocity of $2.1 \mathrm{~km} / \mathrm{s}$. The background velocity is $2.0 \mathrm{~km} / \mathrm{s}$. The true model for the second experiment is shown in Fig. 4a where there are now two heterogeneities each with a velocity of $2.1 \mathrm{~km} / \mathrm{s}$. The background velocity is again $2.0 \mathrm{~km} / \mathrm{s}$. 

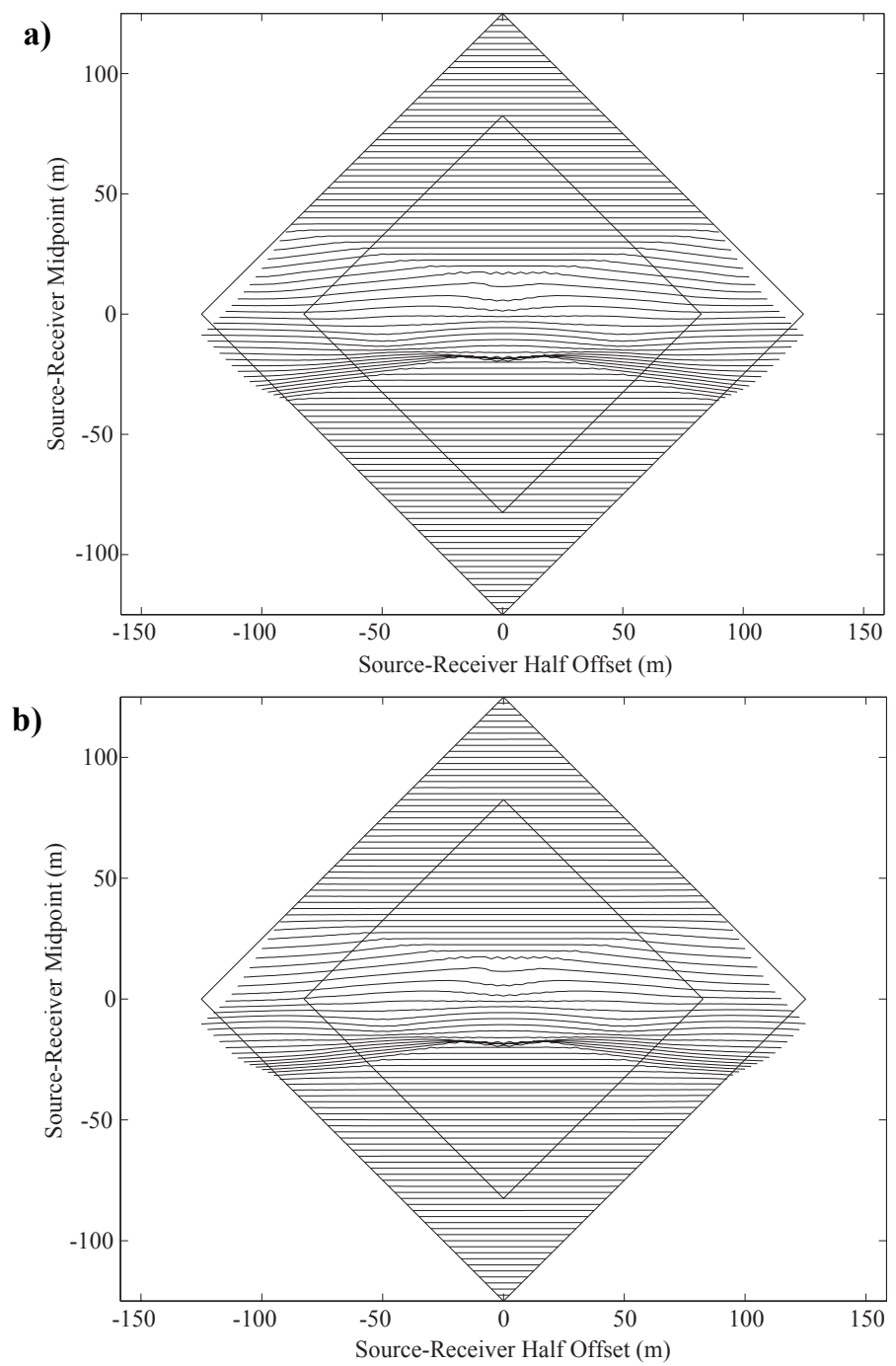

Fig. 2. a) Plot of the full travel-time differences for the single heterogeneity case is shown as a function of source-receiver half offset and source-receiver midpoint in depth. The data within the inner diamond show the partial dataset after removing 10 shots and 10 receivers from the top and bottom of the boreholes. b) Plot of the extrapolated dataset. 


\section{AR EXTRAPOLATION OF THE DATA FOR THE CROSS-BOREHOLE GEOMETRY}

For each model, rays are traced for all possible shot-receiver combinations for the synthetic true model using the code CRT of Červený et al. (1988). Travel-time differences for the first arrivals are then calculated between the true model and starting model, where the starting model is assumed to be homogeneous with a background velocity of $2.0 \mathrm{~km} / \mathrm{s}$. The travel-time data are plotted for source-receiver half offset versus source-receiver midpoint coordinates in depth. With the source and receiver depth coordinates given by $z_{s}$ and $z_{r}$, then the source-receiver half offset is given by $\left(z_{r}-z_{s}\right) / 2$ and the source-receiver midpoint is given by $\left(z_{r}+z_{s}\right) / 2$. This basically takes the travel-time differences plotted on a $\left(z_{s}, z_{r}\right)$ rectangle of source and receiver depth coordinates in the two boreholes and rotating this by 45 degrees. These rotated coordinates have the effect of flattening the travel-time anomalies on the plots. Also, the travel-time anomalies are normalized to the maximum anomaly and plotted on the page along lines.

For the first model with a single heterogeneity, Fig. 2a shows the plot of the full dataset in the outer diamond. The data within the inner rectangle is the partial data after
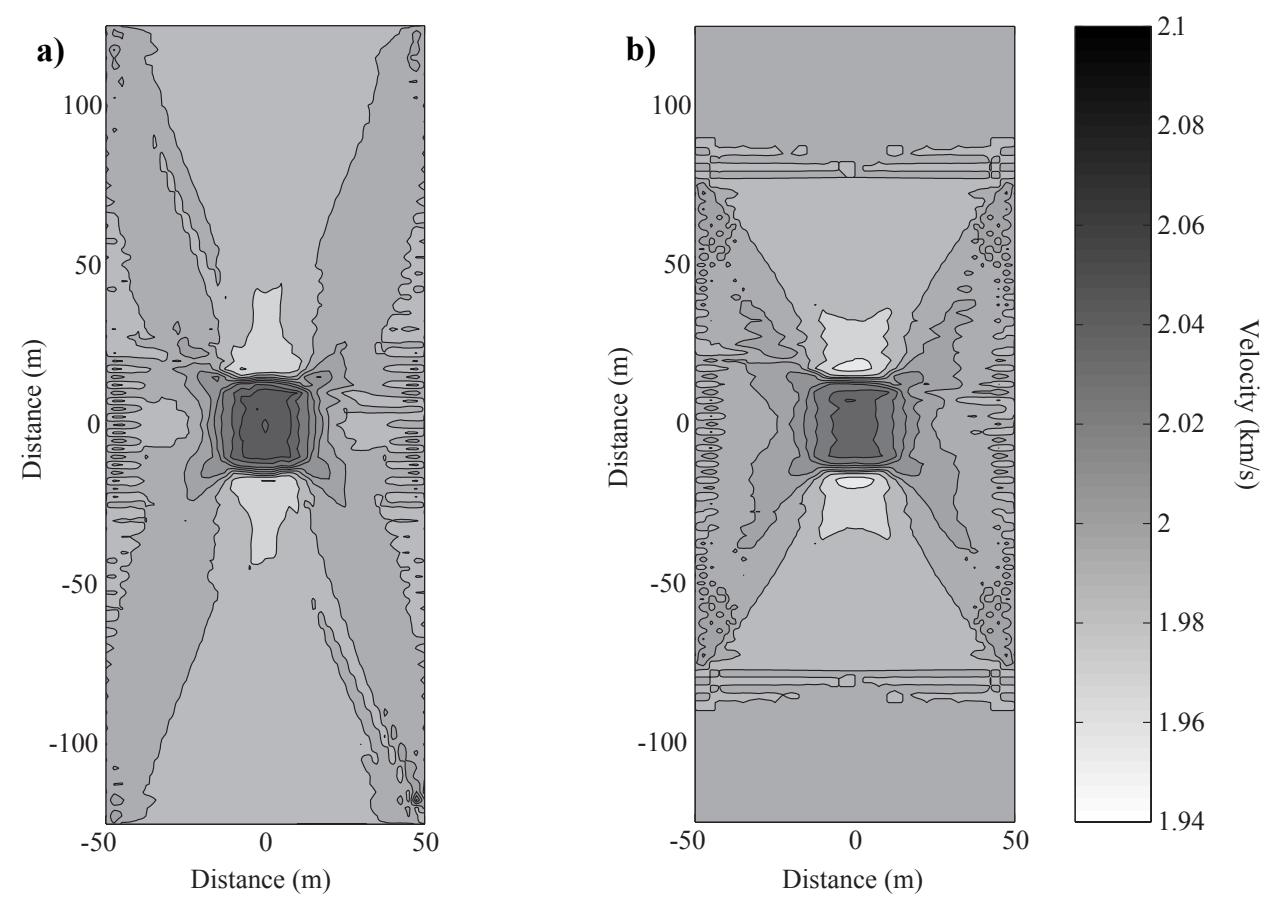

Fig. 3. a) Inversion result with the full dataset for the single heterogeneity case (data within the outer diamond in Fig. 2a). b) Inversion result with the partial dataset (data within the inner diamond in Fig. 2a). 
removing 10 shots and 10 receivers from each end of the model. A two-stage AR extrapolation following the approach described by Li and Nowack (2004) is applied to the partial data within the inner diamond, and the resulting extended dataset is shown in Fig. 2b. Comparing the full dataset in Fig. $2 a$ and the extended dataset in Fig. 2b, the overall pattern is very similar. This shows that the two-stage AR extrapolation technique can work for this synthetic cross-borehole geometry. An important aspect of this source and receiver geometry is that all the heterogeneities are sampled by at least one ray, with the ray limitation mostly related to the angular coverage.

The second model has two heterogeneities and is shown in Fig. 4a. Fig. 4b displays the full dataset of the differences of the first arrival travel-times between the true model and homogeneous starting model in the outer diamond. The travel-time data in the inner diamond shows the partial ray coverage after removing 10 shots and 10 receivers from each end of model. The two stage extrapolation approach of $\mathrm{Li}$ and Nowack (2004) is applied to the partial data and the extended data is shown in the outer diamond in Fig. 4c. This again shows that the two stage autoregressive extrapolation works well for the crossborehole geometry. This case also illustrates the noise reduction inherent in the extrapolation since there is some numerical noise resulting from the choice of accuracy in the ray tracing results for the full travel-times in the outer diamond shown in Fig. $4 \mathrm{~b}$ and this has been reduced by the extrapolation shown in Fig. 4c.
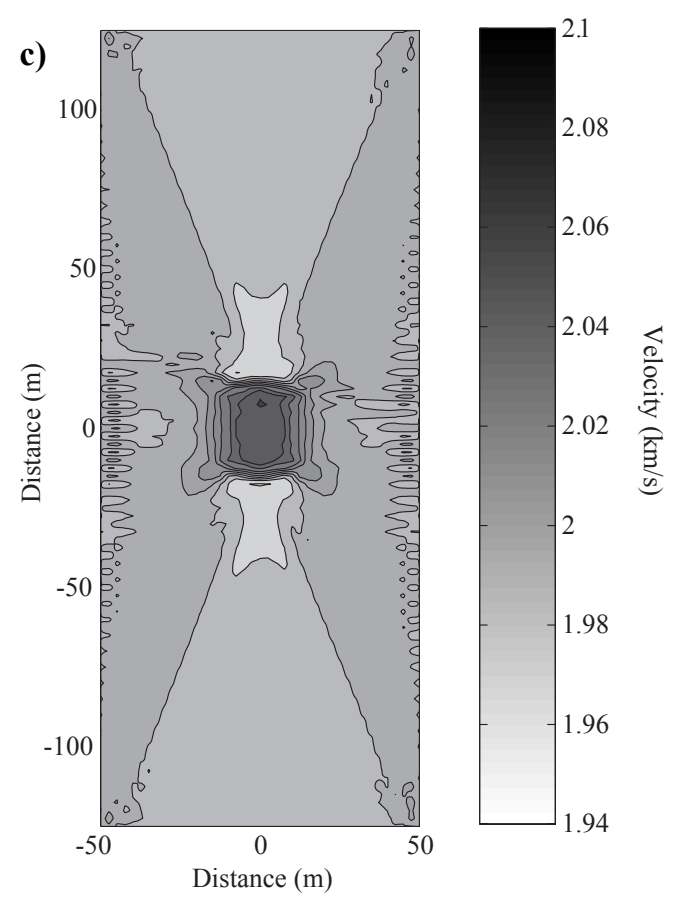

Fig. 3. c) Inversion result with the extended dataset (data within the outer diamond in Fig. 2b). 

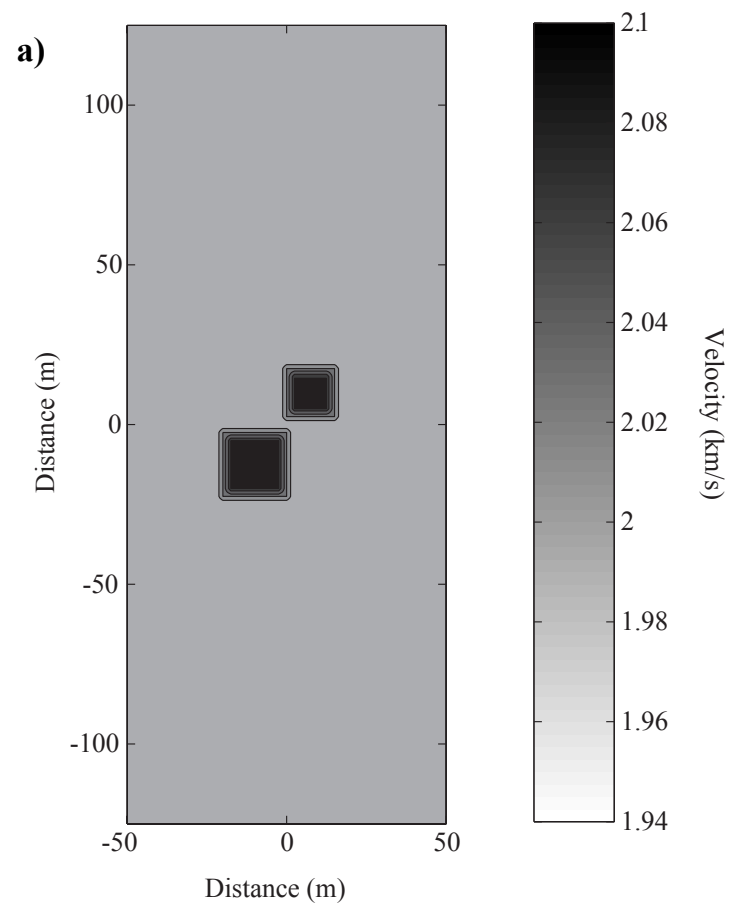

Fig. 4. a) True model for the two heterogeneity case.

\section{TOMOGRAPHIC INVERSIONS USING FULL, PARTIAL, AND EXTENDED DATASETS}

The full dataset for the single heterogeneity shown in Fig. 2a, the partial dataset within the inner diamond in Fig. 2a, and the extended dataset shown in Fig. 2b are all used for regularized, damped tomographic inversions following Li and Nowack (2004) (see also, Tarantola, 2005; Wang, 1993). The full dataset for the two heterogeneity case is shown in Fig. $4 \mathrm{~b}$ with the extrapolated data in Fig. 4c.

Figs. $3 a-3 c$ demonstrate the tomographic reconstructions of the single heterogeneity case for full, partial and extended datasets, respectively. Comparing these tomographic inversions, we see that all three of them have similar vertical resolutions. However, Figs. $3 \mathrm{a}$ and $3 \mathrm{c}$ have much better horizontal resolutions than Fig. $3 \mathrm{~b}$ which has larger side lobe. The better horizontal resolution for Fig. 3a results from more oblique rays for the full dataset in Fig. 2a that better constrains the positions of the sides of the high velocity heterogeneity (Menke, 1984). The inversion result with the extended data shown in Fig. 3c is very close to the inversion using the full dataset in Fig. 3a, and greatly improves the horizontal resolution from the partial data reconstruction in Fig. 3b. This is not surprising since the extrapolated travel-time differences in Fig. $2 b$ are very similar to the full dataset 

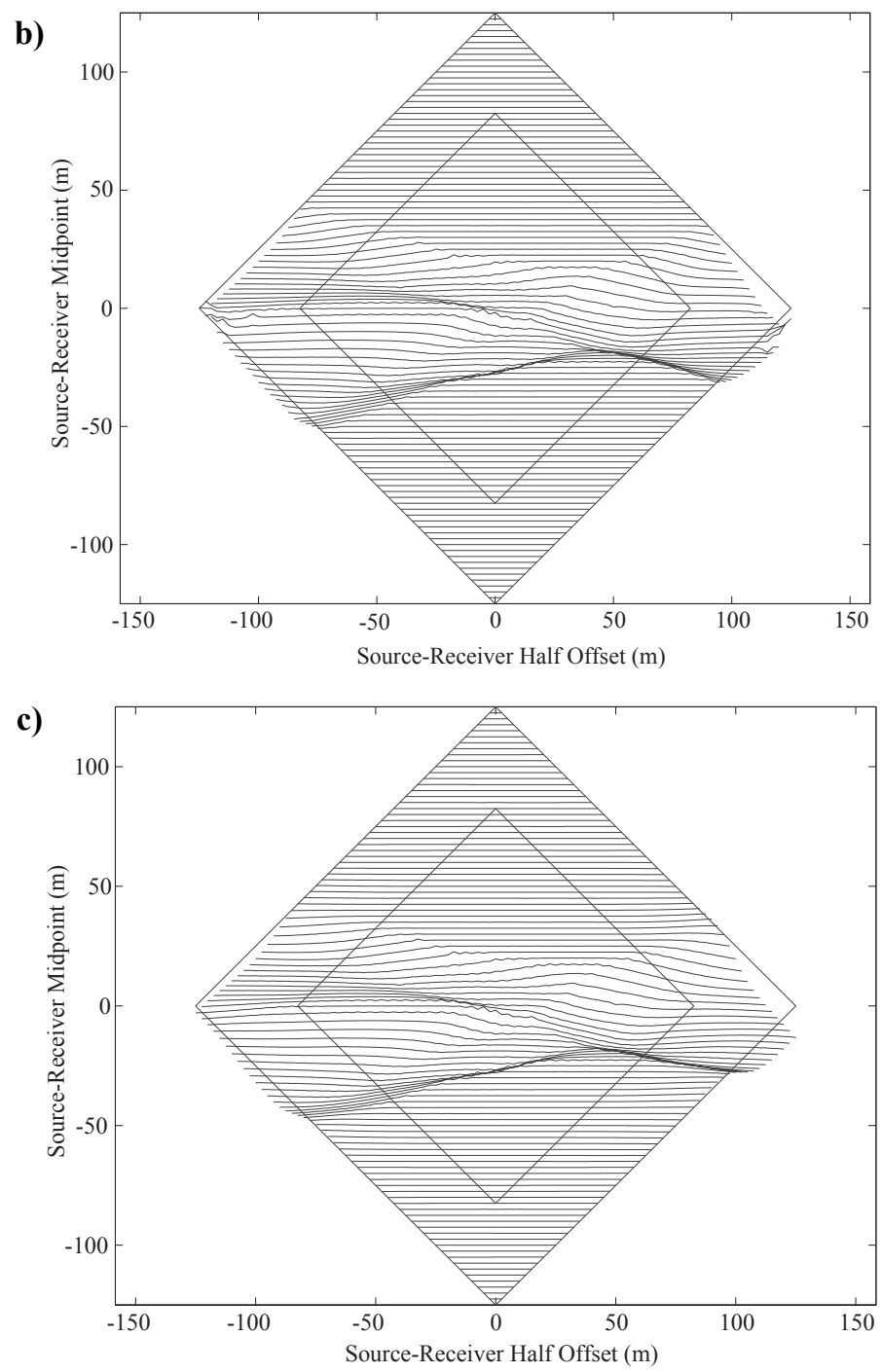

Fig. 4. Plots of the full travel-time differences for the two heterogeneity case. b) Plot of the full dataset, the data within the inner diamond show the partial dataset after removing 10 shots and 10 receivers from the top and bottom of the boreholes. c) Plot of the extrapolated dataset.

in Fig. 2a. But it shows that the two-stage AR extrapolation method can efficiently extrapolate the partial travel-time data to a broader range for the cross-borehole tomography geometry, and it can be an effective way to partially overcome the limitation of ray coverage for real cross-borehole tomographic experiments. Note however that for inversion, the data extrapolation is being used as an additional regularization on the reconstruction of the velocity. 

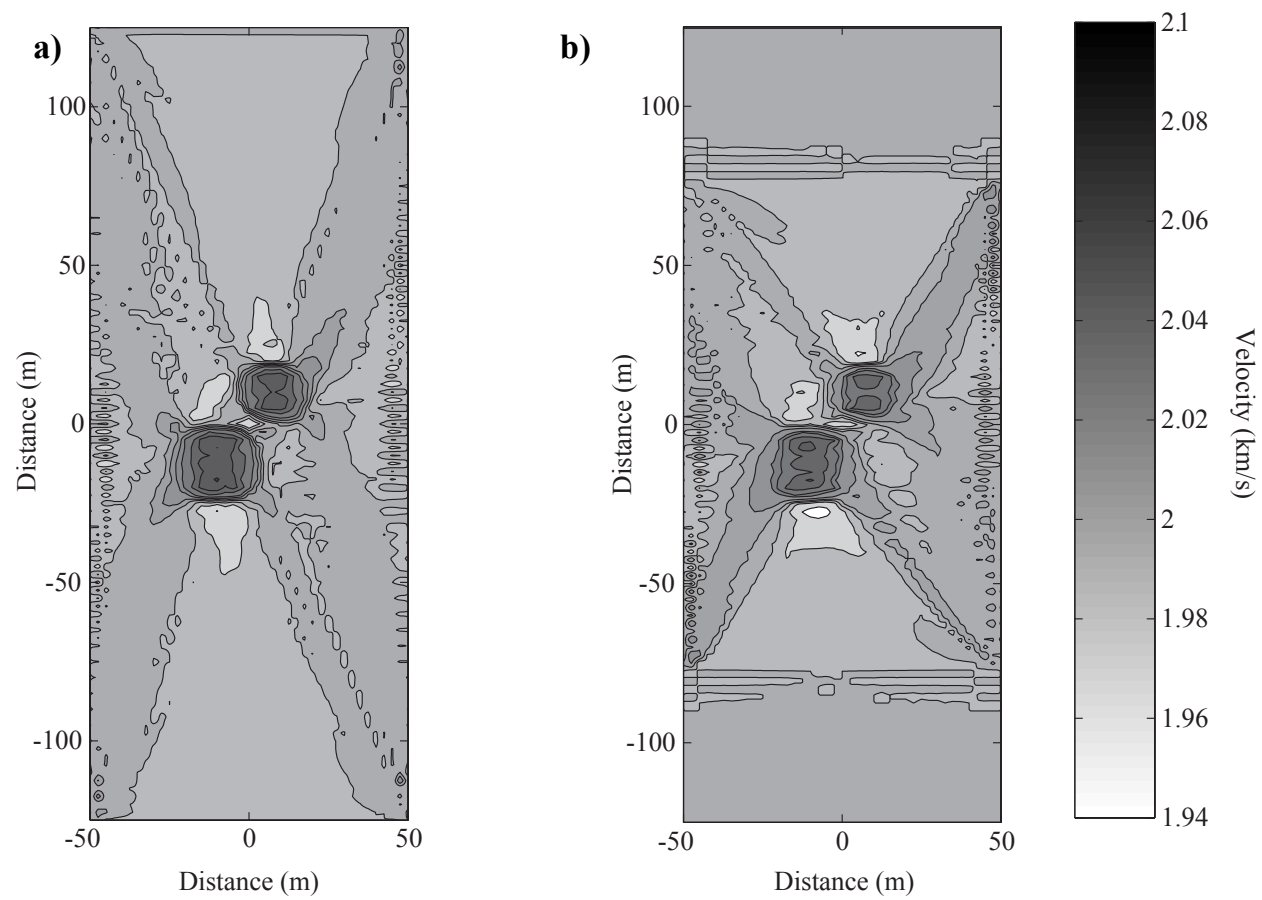

Fig. 5. a) Inversion result with the full dataset for the two heterogeneity case (data within the outer diamond in Fig. 4b). b) Inversion result with the partial dataset (data within the inner diamond in Fig. 4b).

For the two heterogeneity case, the tomographic reconstructions using the full data, the partial data and the extrapolated data are shown in Figs. 5a, 5b and 5c, respectively. For this case the side lobes are also larger for the partial data case. Also, the inverted anomalies in the partial data case are somewhat more subdued than for the inversion using the complete dataset. The inversion results for the extended data shown in Fig. 5c are very similar to the inversion result using the full dataset in Fig. 5a. Again, this is directly related to the good extrapolation results of the travel-time data using the AR approach in Fig. 4c. The extended data inversion is even slightly better than the full data case since some of the slight numerical noise in the full data have been eliminated in the extrapolation process. This example shows that the AR extrapolation can be applied to the tomographic inversion for a several heterogeneity case using the cross-borehole geometry.

\section{CONCLUSIONS}

The two-stage AR extrapolation technique has been shown by Li and Nowack (2004) to be an effective way to perform data extrapolation of travel-times for seismic tomography using laboratory geometries. To investigate whether the two-stage AR extrapolation algorithm improves the tomographic results for a cross-borehole geometry, 

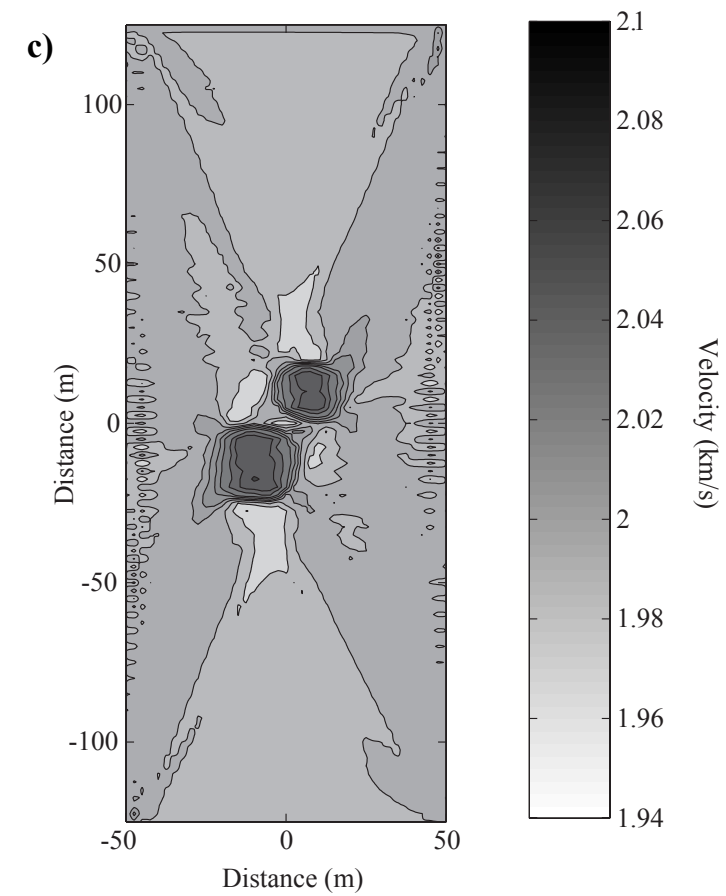

Fig. 5. c) Inversion result with the extended dataset (data within the outer diamond in Fig. 4c).

tests on synthetic data were performed. Both the extrapolated travel-time data and the tomographic reconstructions with the extrapolated data show that AR extrapolation can effectively extend the synthetic cross-borehole travel-time data to a broader coverage and can improve the tomographic reconstruction results using cross-borehole geometries.

In order to apply this technique to seismic tomography, there must be adequate traveltime coverage in order to construct a travel-time surface that can be extrapolated. Also, a sufficient amount of the pattern in the travel-time anomalies must exist in the observed data so that a reasonable extrapolation can be performed. If there is random noise in the observed data, then the extrapolated data will emphasize the more predictable parts of the data (Li and Nowack, 2004). Nonetheless, any coherent noise in the data will also be extrapolated. For complicated observed data, further study is required on what the best size of the prediction error filter is required to extrapolate the data.

The results shown here are for a linearized tomography problem for small velocity contrasts and first arrival data. For a nonlinear tomography problem, the autoregressive extrapolation would need to be applied at each linearized step since the travel-time residuals will change. It is unknown what the convergence properties of such an algorithm would be. Also, for media with larger contrasts triplicatations of the travel-time data will result. For this case, AR extrapolation might be applied to data that have been processed to unwrap the triplications such as by tau-p or slant stack processing methods (Gardner and $\mathrm{Lu}, 1991)$. 
AR extrapolation could have applications to seismic tomography experiments with similar restrictions on the ray coverage, such as the teleseismic tomography problem where a passive seismic array at the surface is used to record travel-times from teleseismic waves coming from below the structure. The teleseismic tomographic approach is sometimes referred to as the $\mathrm{ACH}$ inversion method after Aki, Christoffersson and Husebye (Iyer and Hirahara, 1993; Aki and Richards, 1980). In the cross-borehole and $\mathrm{ACH}$ geometries, the velocity heterogeneities are always sampled by either horizontal or near vertical rays, respectively, but the angular ray coverage is limited. Thus, AR extrapolation as described here may be applicable to a number of similar experimental geometries in order to extend the effective angular data coverage. Nonetheless, the primary usage here for the AR extrapolation of the data is for the regularization of the partial coverage, tomography problem.

\section{References}

Aki K. and Richards P., 1980. Quantitative Seismology. Freeman, San Francisco, USA.

Červený V., Klimeš L. and Pšenčík I., 1988. Complete seismic ray tracing in three-dimensional structure. In: K.J. Doorknobs (Ed.), Seismological Algorithms. Academic Press, San Diego, CA, USA, 89-168.

Claerbout J.F., 1992. Earth Soundings Analysis: Processing versus Inversion. Blackwell Scientific Publications, Cambridge, Massachusetts, USA.

Claerbout J.F., 1998. Multidimensional recursive filters via a helix. Geophysics, 63, 1532-1541.

Claerbout J.F. and Fomel S., 2005. Geophysical Estimation by Example: Geophysical Soundings Image Construction: Multidimensional Autoregression. http://sepwww.stanford.edu /sep/prof/index.html

Fomel S. and Claerbout J.F., 2003. Multidimensional recursive filter preconditioning in geophysical estimation problems. Geophysics, 68, 577-588.

Iyer H.M. and Hirahara K., 1993. Seismic Tomography Theory and Practice. Chapman Hall, London,U.K..

Li C. and Nowack R.L., 2004. Application of autoregressive extrapolation to seismic tomography. Bull. Seismol. Soc. Amer., 94, 1456-1466.

Li C. and Nowack R.L., 2005. Seismic tomography using travel-time surfaces for experiments in the laboratory. J. Geophysics and Engineering, 2, 231-237.

Lu L. and Gardner G.H.F. (Eds.), 1991. Slant-Stack Processing. Society of Exploration Geophysicists, Tulsa, OK, USA.

Menke W., 1984. The resolving power of cross-borehole tomography. Geophys. Res. Lett., 11, $105-108$.

Tarantola A., 2005. Inverse Problem Theory and Methods for Model Parameter Estimation. Society for Industrial and Applied Mathematics, Philadelphia, USA.

Wang B., 1993. Improvement of Seismic Travel-time Inversion Methods and Application to Observed Data. PhD Thesis, Purdue University, West Lafayette, IN, USA. 